\title{
A Generative Approach for Die Pattern Machining
}

\author{
Hayong Shin, Robert M. Trecapelli, Andrew Shudra Jr., Yun C. Chung \\ CAD/CAE/CAM R\&D, Techical Computing Center, Chrysler Corp.
}

Key words: lost foam pattern, feature recognition, generative process planning

Abstract: Manufacturing a press die for car body panel usually starts from making a Styrofoam pattern (for short, 'pattern') for lost wax casting process. Industry trend is to build patterns by NC machining. Since the mating planes for assembly or die face of casting stock are to be machined later, pattern should have some stock allowance on those surfaces, which can be as large as $12 \mathrm{~mm}$. That means pattern itself can have fairly large amount of machining tolerance compared to die machining. Styrofoam is easy to cut, but fragile. Hence, the process planning for pattern machining should reflect some different aspects than that of die machining. In this paper, presented is the generative approach taken by Chrysler to create tool path to cut pattern from the CAD model of die. The approach includes steps of 1) feature recognition, 2) automatic process planning and 3) tool path generation.

\section{INTRODUCTION}

Usually major components of a stamping die are cut from the cast raw stocks, which are typically cast by use of lost wax process. Fig. 1 shows the overall process for stamping die manufacturing. The top layer of Fig. 1 shows the information flow, the middle layer shows sequential activities, and the bottom layer depicts physical materials. This paper is focused on the "pattern building" activity, the second box of the activity layer in Fig.1, which is to build a Styrofoam pattern (or just pattern for short) from the die model. Traditionally, pattern is built in manual manner while die design is done in paper drawing form. Recently, the technique of solid modeling is being widely used in die design, NC machining is becoming a strong alternative for pattern building.

Fig.2 describes the detail process to build pattern by $\mathrm{NC}$ machining from the die model. Again, this paper is focused on the "NC programming" activity, the third box of the activity layer in Fig.2. The first step is to create the pattern model out of the die model by adding extra stock allowance on each face to be machined in die machining activity of Fig.1. Those faces include mating planes and die face. The amount of the stock allowance depends on the precision of casting process, and is usually around $1 / 2$ inch. Hence pattern machining may take a fairly big tolerance compared to that of die machining.

The second step is to split the pattern model into slices that can be machined. The criteria for the location to split are 1) to minimize the number of slices, 2) to make the hidden features accessible by the 3 axis machine, 3 ) to fit in the limit of tool length and merchantable Styrofoam raw stock size, and 4) to avoid thin slice which can be broken during machining. For example, the punch model in Fig.3a can be built by 3 axis NC machining after slicing into 2 pieces as in Fig.3b. Usually, a major component of drawing dies, such as punch, punch shoe, die, and die shoe, is split into 2 4 slices.

The next two steps are to generate tool path and to cut each slice. Since Styrofoam is easy to cut but brittle, required is a different kind of process planning strategy than that of die machining. These steps will be elaborated in the following sections.

After making slices by NC machining, the final step is to assemble the slices to make a pattern. In this step, pattern maker does a lot of jobs including, but not limited to, gluing the slices, making chamfers with the special chamfering tool, and filling gaps between slices if any.

The rest of this paper will be devoted to NC programming for pattern machining. General purpose CAM system can be used for this job, but it is not efficient. Thus we developed a

The original version of this chapter was revised: The copyright line was incorrect. This has been corrected. The Erratum to this chapter is available at DOI: 10.1007/978-0-387-35392-0_40 
dedicated and fully automatic CAM system, called GPM(Generative Pattern Machining). Section 2 will describe the architecture of GPM, and in section 3 and 4 its key modules will be explained, followed by some examples and conclusion.

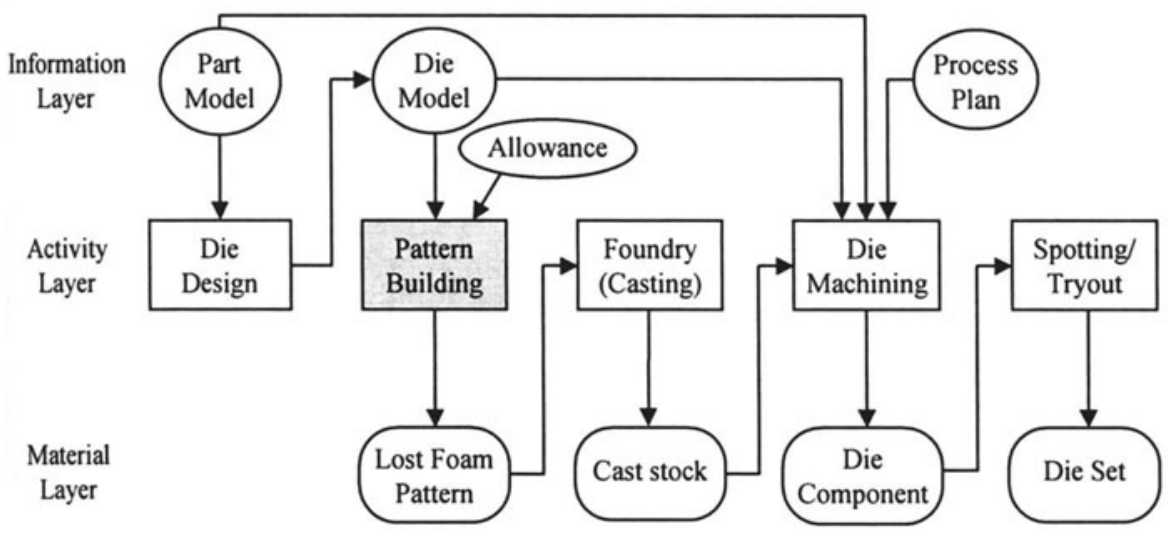

Figure 1. Die manufacturing process

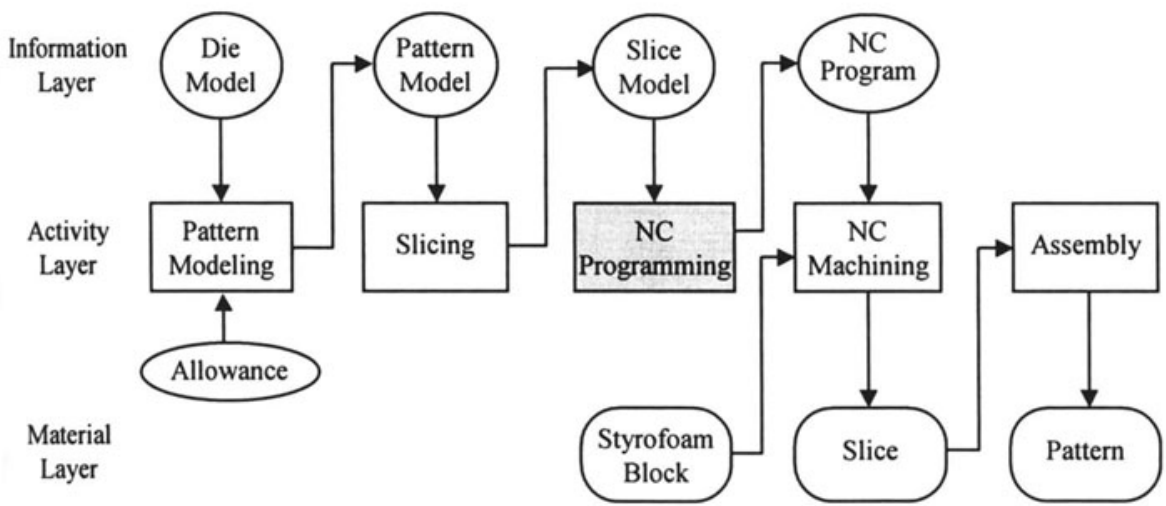

Figure 2. Pattern building process

\section{ARCHITECTURE OF GPM}

The purpose of GPM is to provide pattern makers with a handy tool which requires very little user interaction. GPM consists of two separate programs. One is the direct interface functions embedded in CATIA, which exports the part surface model and slice model of pattern into external file. The other is main GPM program, which reads the exported files in and computes the tool paths. The main GPM program is invoked by embedded interface function. In turn, GPM is made of 4 modules, namely 1) Z-map model construction, 2) feature recognition, 3 ) process planning, and 4) tool path generation. The latter 3 modules will be explained in the following sections.

One of the reasons for exporting part surface is to help the pattern makers to add stock allowance for the part surface. Unlike the planar mating planes, the part surface (such as punch surface) is a very complex sculptured surface, and hence it is not easy to offset in usual manner. Hence we allow the user to leave adding stock allowance for the part surface to be done by GPM later. 


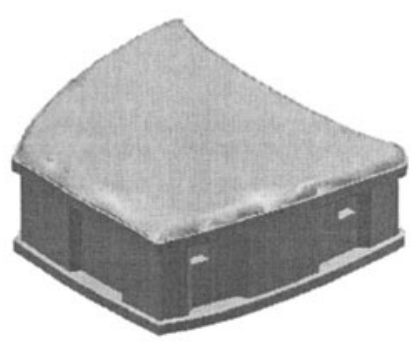

(a) Punch

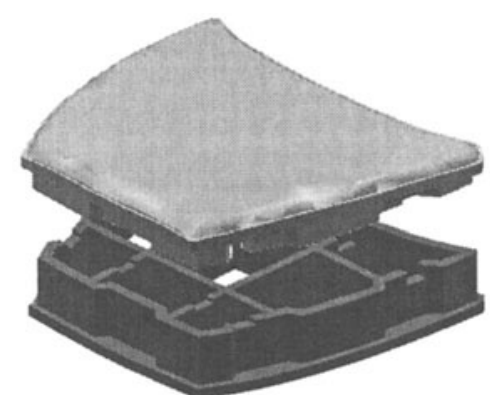

(b) Sliced models

Figure 3. Punch split into 2 slices

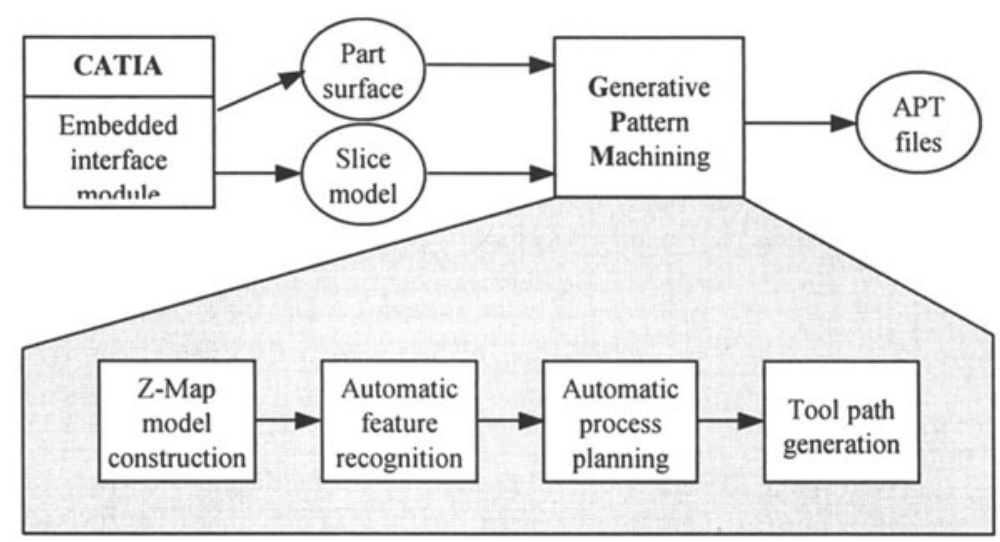

Figure 4. Architecture of GPM

Most of the slice models need two side machining, once from the top, and once from the bottom, and usually the bottom side needs to be machined first, especially when the top side has a sculptured surface. Otherwise, the setup operation for the bottom side machining becomes extremely hard. The 4 steps of GPM in Fig. 4 is to be applied twice (bottom and top) for each slice.

Since GPM converts the model into Z-map representation [1], the offsetting operation becomes easier [2]. Z-map is a discrete and approximate representation scheme storing the Zvalues of the model over a grid. The approximation error of Z-map scheme depends on the grid interval. As the grid interval becomes smaller, the approximation error becomes small, but more memory and computation time are required. Since the pattern machining has a big machining tolerance as mentioned above, usually $1 \mathrm{~mm}$ of grid interval gives sufficient accuracy as well as acceptable computation time and memory requirement.

Construction of Z-map can be done easily. First, read the part surface model and make zmap model out of it by triangulation of the part surface and evaluation of z-value at each grid point. Then, offset the z-map model by the stock allowance. Then read the slice model, which is polyhedral solid model, and update the z-map model by each face.

\section{FEATURE RECOGNITION}

In order to generate $\mathrm{NC}$ code from $\mathrm{CAD}$ data in automatic manner, machining feature recognition together with automatic process planning is an essential step. Various types of approach to feature recognition have been reported in the literature $[4,5,6,7]$. For the 
comprehensive survey, the readers are referred to [7]. In this paper, feature recognition is performed in two phase manner, namely, 1) machining region identification, and 2) feature type classification.

\subsection{Machining region identification}

The first phase is to identify regions which need to be machined. According to the classification of machining region recognition methods described in [7], a sectioning method is used in GPM since it goes well with Z-map representation. After sectioning Z-map model by a series of planes parallel to XY plane, each Z-map grid point is marked by the layer number. Adjacent grids having same layer number are grouped to form a micro region. Adjacent micro regions having similar characteristics are merged to form a macro region. The macro regions are machining regions, except the top most regions coincident with the splitting plane which is obtained from the Styrofoam raw stock.

\subsection{Feature type classification}

The regions identified in the previous step merely show where to machine. In order to determine how to machine them, those regions identified are to be classified. In other words, the type of machining region is required to determine the machining sequence and the tool path type. In this application, the types of feature are predefined as follows (see Fig.5) :

a) Periphery : outer boundary of the slice

b) Through hole : bottomless hole inside the periphery

c) Pocket : a machining region with curved or planar bottom

c-1) Closed pocket : a region totally surrounded by higher regions

c-2) Partially open pocket : a pocket region with lower and higher neighbour

d) Roof (totally open pocket) : a region that all its neighbour are lower than itself

e) Special features

e-1) Bolt slot : slot for a mounting bolt

e-2) feature on a rib : slot or step on a rib

It is worth mentioning that the shapes of special features are not much different from the others, but the location where the feature resides determines the special type features. For example, a slot on a rib looks like an partially open pocket, except that it is on a rib. The reason for differentiating special features is because they should be treated with care when process planning.

\section{PROCESS PLANNING AND TOOL PATH GENERATION}

Generally speaking, the process planning means 1) assigning a tool path type to a feature, 2) determining the cutting tool and parameters such as tool path interval, and 3) sequencing the machining features. In this application, the tool path type is determined by the type of feature as depicted in Table 1 and Fig.6, and the cutting tool is fixed as a $50 \mathrm{~mm}$ ball end mill. Though the cutting load on the cutter or the machine hardly matters, the tool path interval is determined by compromising the machining time versus the cusp height, and from the experience it is set to $8 \mathrm{~mm}$.

Since Styrofoam is fragile, it is very important to have a proper machining sequence. In usual machining application, finishing after roughing is good for the machined surface quality. However, it is not a good idea for this application, because roughing will weaken the support structure such as a rib, which will make it wobble while being finished. It may result in a bad surface quality or a broken rib in even worse case. 


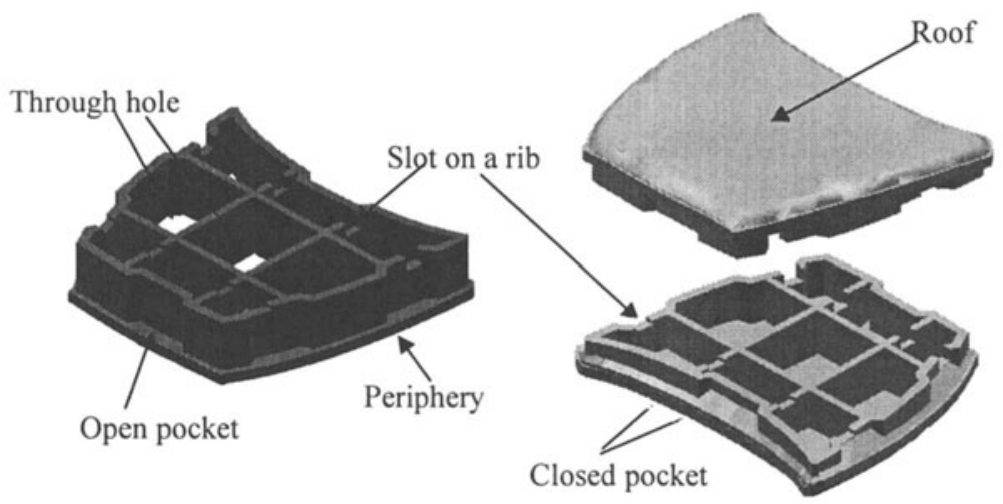

Figure 5. Types of features

Table 1. Feature type and corresponding tool path type (DW: $\rightarrow 6$ )

\begin{tabular}{|c|c||c|c|}
\hline \multicolumn{2}{|c||}{ Feature type } & Priority & Tool path \\
\hline \hline \multicolumn{2}{|c|}{ Periphery } & 2 & profiling \\
\hline \multicolumn{2}{|c|}{ Through hole } & 2 & profiling \\
\hline \multirow{2}{*}{ Pocket } & Closed pocket & 3 & contour parallel pocketing \\
\cline { 2 - 4 } & Partially open pocket & 3 & direction parallel scanning \\
\hline \multicolumn{2}{|c|}{ Roof (totally open pocket) } & 3 & direction parallel scanning \\
\hline \multirow{2}{*}{$\begin{array}{c}\text { Special } \\
\text { feature }\end{array}$} & Bolt slot & 2 & profiling \\
\cline { 2 - 4 } & Step/Slot on a rib & 1 & direction parallel scanning \\
\hline
\end{tabular}

The machining sequence is determined by giving priorities to the feature types. For example, since a rib does not have enough strength, the features on a rib should be machined first, even before the rib is formed out of the block. The priorities for each feature types are shown in Table 1, meaning that the features with priority number 1 are to be machined first, then priority 2 , and so on. The features with the same priority are ordered by solving travelling salesperson problem (TSP). In our implementation, a simple greedy-type algorithm is used, which may give the result far from the optimal solution.

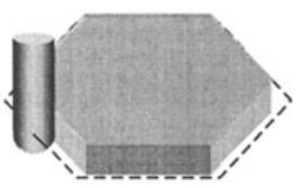

(a) profiling

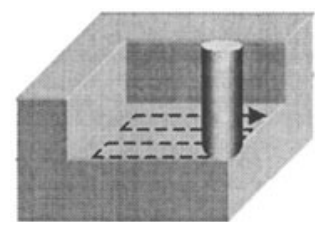

(b) Direction parallel scanning

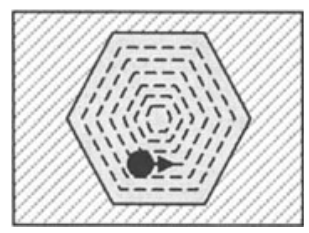

(c) Contour parallel pocketing

Figure 6. Tool path types

Periphery feature and through hole feature have the same tool path type (profiling) and the same priority. In most case, the profiling operations for those features are to be done from the top side. Especially, for the slice model with more than one lump, the profiling operation should be done from the top side after machining the bottom side in order that the lumps 
remain in a single piece of Styrofoam block. When the row stock height of Styrofoam block is bigger than the effective tool length, the profiling operations can be done by half way depth from both side with a little overlap as depicted in Fig.7.

With the process plan, tool path generation is an easy part. After obtaining the boundary curve of the machining region, the actual tool paths for features are computed according to the tool path type as shown in Table 1 and Fig.6. As for the detail algorithm for generating pocketing, scanning, and profiling tool paths, the readers are referred to [3]. Profiling tool paths are obtained by offsetting the boundary curve. Voronoi diagram can be used to get contour parallel pocketing tool paths.

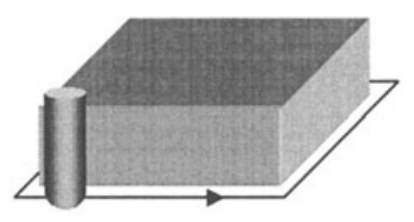

(a) Full depth profiling

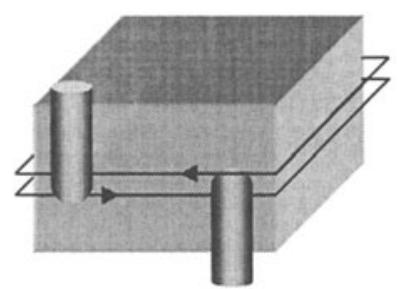

(b) Half depth profiling

Figure 7. Profiling operation

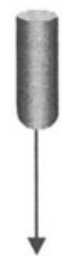

(a) Plunging approach

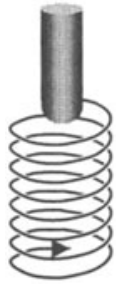

(b) Spiral approach

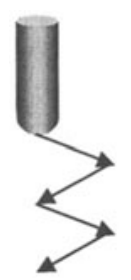

(c) Sawtooth approach

Figure 8. Approach motions

Special attention should be paid to the approach motion. With a plunging approach motion, evacuation of the Styrofoam chips becomes a problem causing particles to melt together loading the hollow cutter. Instead, the spiral approach motion is used as much as possible. If the spiral motion is not available due to the space limitation in the approach area, the saw tooth approach is used, which is always possible. Fig. 8 shows these three approach motions.

\section{EXAMPLES}

Fig.9 is a lower shoe model, Fig.10 shows the features recognised from it, and Fig.11 reveals the tool paths for it. 


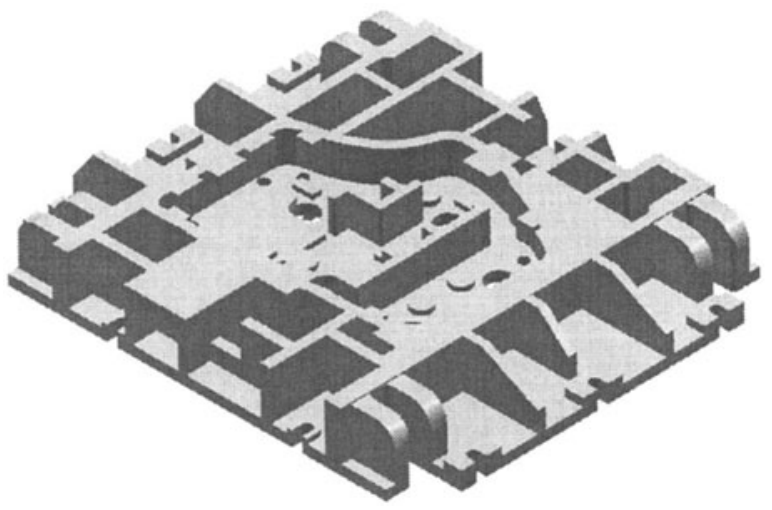

Figure 9. CATIA model of a lower shoe

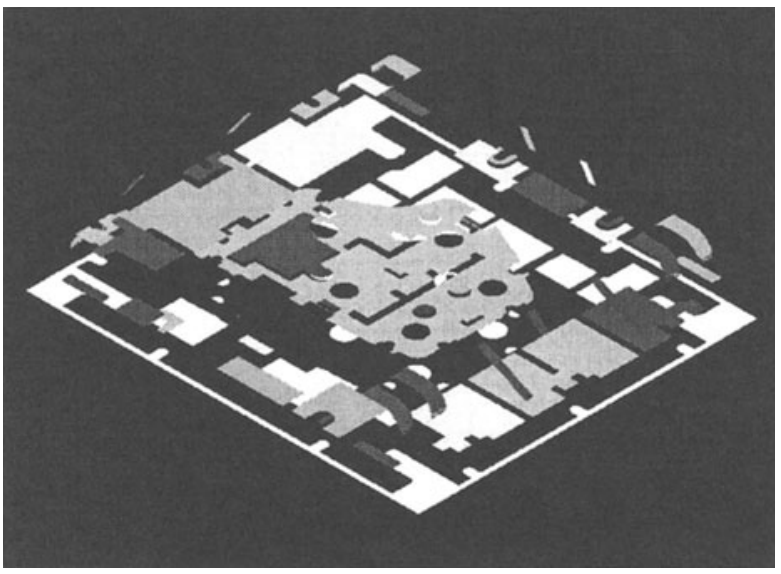

Figure 10. Features from Fig.9

\section{CONCLUDING REMARKS}

In this paper, we have shown the overall architecture of the software and details of the GPM (Generative Pattern Machining) system, which is in pilot at Chrysler Corporations Pattern Shop.

GPM is a good example of CAPP/CAM integrated system. Previously die pattern programming and machining required a high amount of skill with CAD system and CNC machining was not always optimized. The objective was to lessen the amount of user interaction with both CATIA and third part software, limit the geometry creation needs by the user, and use best practices for machining die patterns. 


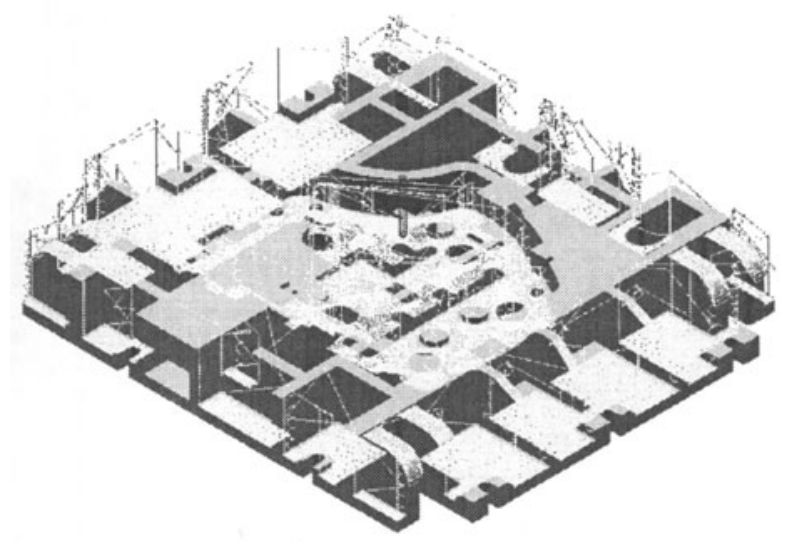

Figure 11. Tool paths for the lower shoe in Fig.9

\section{REFERENCES}

[1] Choi, B K, Surface Modeling for CAD/CAM, 1991, Elsevier, Amsterdam $(9 \rightarrow 8)$

[2] Choi, B.K., Chung, Y.C., Park, J.W. and Kim, D.H., "Unified CAM system architecture for die and mold manufacturing", Computer-Aided Design, V26, N3, 1994, pp.235-243

[3] Held, M, On the Computational Geometry of Pocket Machining, Springer-Verlag, 1991

[4] Joshi, S and T C Chang, "Graph-based heuristics for recognition of machined features from a 3D solid model", Computer-Aided Design, V20, N2, 1988, pp.58-66

[5] Kim, Y S, "Recognition of form features using convex decomposition", Computer-Aided Design, V24, N9, 1992, pp.461-476

[6] Sakurai, H and D C Gossard, "Recognizing shape features in solid models", IEEE Comp. Gr. \& Appl., V10., N5, 1990, pp.22-32

[7] Shah, J and M.Mantyla, Parametric and Feature-based CAD/CAM : concepts, techniques, and applications, 1995, John Wiley \& Sons, NY 